PROCEEDINGS OF THE

AMERICAN MATHEMATICAL SOCIETY

Volume 141, Number 9, September 2013, Pages 2945-2950

S 0002-9939(2013)11558-3

Article electronically published on May 7, 2013

\title{
THE EISENSTEIN FAMILY
}

\author{
ROBERT F. COLEMAN
}

(Communicated by Lev Borisov)

Abstract. Based on the work of Buzzard, Kilford, and Roe, we state a general conjecture about the family of overconvergent Eisenstein series.

In [CM98, Mazur and Coleman defined a rigid analytic object called the eigencurve. This object parametrizes finite slope overconvergent normalized $p$-adic eigenforms. The Eisenstein family is a family of overconvergent modular forms whose existence was essential for this construction.

The geometry of the eigencurve is still poorly understood. In [BK05], when $p=2$ (a case not actually dealt with in BK05], but see Bu07]), it was proven that the 2-adic level one eigencurve at the "boundary of weight space" is a disjoint union of infinitely many annuli. This has been extended to the 3 -adic level one eigencurve in Ro09,1 While it might be too optimistic to expect this feature to be possessed by many more eigencurves, we believe results obtained and used in BK05] and Ro09. about the domain of analyticity of the Eisenstein family will generalize. In this note, we reformulate these results and state a general conjecture.

\section{THE FAMILY}

Let $\mathbf{q}=4$ if $p=2$ and $p$ otherwise. Let $\mathbf{C}_{p}$ be the completion of an algebraic closure of $\mathbf{Q}_{p}, \mathbf{R}_{p}$ the ring of integers of $\mathbf{C}_{p}$ and $\mathbf{F}$ the residue field of $\mathbf{R}_{p}$. Let $\mathbf{W} \subset \mathbf{R}_{p}$ denote the Witt vectors of $\mathbf{F}$. Also let $v$ denote the valuation on $\mathbf{C}_{p}^{*}$ such that $v(p)=1.2$ Let $Z$ be the connected component of the ordinary locus of $X_{1}(\mathbf{q})$ containing the cusp $\infty$. Let $\mathcal{W}$ be the analytic group of continuous $\mathbf{C}_{p^{-}}$ valued characters on $\mathbf{Z}_{p}^{*}$ and $\mathcal{B}$ the subgroup of characters trivial on $\mu\left(\mathbf{Q}_{p}\right)$. Let $\boldsymbol{\Lambda}=\mathbf{Z}_{p}\left[\left[\mathbf{Z}_{p}^{*}\right]\right], \Lambda=\mathbf{Z}_{p}\left[\left[1+\mathbf{q} \mathbf{Z}_{p}\right]\right] \subset \boldsymbol{\Lambda}$, and for $a \in \mathbf{Z}_{p}^{*}$, let $[a]$ denote its image in $\boldsymbol{\Lambda}$. If $\kappa \in \mathcal{W}$ and $\alpha=r_{1}\left[a_{1}\right]+\cdots+r_{n}\left[a_{n}\right] \in \mathbf{Z}_{p}\left[\mathbf{Z}_{p}^{*}\right]$, let

$$
\kappa(\alpha)=\sum_{i=1}^{n} r_{i} \kappa\left(a_{i}\right) .
$$

This extends to a continuous homomorphism $\Lambda \rightarrow \mathbf{R}_{p}$ and induces a homomorphism

$$
\kappa: \Lambda[[q]] \rightarrow \mathbf{R}_{p}[[q]]
$$

Let $w_{p}$ be the function on $\mathcal{W}, \kappa \mapsto \kappa([1+\mathbf{q}]-1)$.

Received by the editors January 11, 2010 and, in revised form, March 30, 2011 and November 13, 2011.

2010 Mathematics Subject Classification. Primary 11F33, 11F80.

${ }^{1}$ See BC06] for another result about the geometry of the 2-adic eigencurve.

${ }^{2}$ We will extend $v$ to $\mathbf{C}_{p} \cup\{\infty\}$ and let $v(0)=\infty$ and $v(\infty)=-\infty$.

(C)2013 American Mathematical Society Reverts to public domain 28 years from publication 
Let $\mathbf{E}(q) \in \Lambda[[q]]$ be such that

$$
\kappa(\mathbf{E}(q))=1+\frac{2}{\zeta^{*}(\kappa)} \sum_{n \geq 1} \sigma_{n}(\kappa) q^{n},
$$

for $\kappa \in \mathcal{B} \backslash\{\mathbf{1}\}$, where $\zeta^{*}$ is the $p$-adic zeta function on $\mathcal{W}$ (see Chapter 4 , $\S 3$, of La78) and

$$
\sigma_{n}(\kappa)=\sum_{\substack{d \mid n \\(d, p)=1}} \kappa(d) / d .
$$

If $F(q)=\sum_{n \geq 0} a_{n} q^{n}$ is a series in $q$, we set $V(F)(q)=\sum_{n \geq 0} a_{n} q^{p n}$. By Corollary 2.1.1 of [Co97ii], there is an element $\mathcal{E}_{p} \in A^{\dagger}\left(Z_{\mathcal{B}} / \mathcal{B}\right)^{0}$, the ring of rigid analytic functions bounded by one on $Z_{\mathcal{B}}:=Z \times \mathcal{B}$ overconvergent over $\mathcal{B}$, whose $q$-expansion, $\mathcal{E}_{p}(q)$, is $\mathbf{E}(q) / V(\mathbf{E})(q) \stackrel{3}{3}$

Let $H$ denote the level one, weight $p-1$, Hasse invariant modular form over $\mathbf{F}_{p}$ 出 If $E$ is an elliptic curve over $\mathbf{R}_{p}$, let

$$
h(E)= \begin{cases}v(A) & \text { if } H(\tilde{E}, \tilde{\omega}) \neq 0, A \in \mathbf{R}_{p} \text { and } H(\tilde{E}, \tilde{\omega})=A \bmod p, \\ 1 & \text { if } H(\tilde{E}, \tilde{\omega})=0,\end{cases}
$$

where $\omega$ generates $\Omega_{E / \mathbf{R}_{p}}^{1}, \tilde{E}$ is $E \bmod p$ and $\tilde{\omega}$ is $\omega \bmod p$. (This is independent of the choice of $\omega$.) If $P \in X_{1}(N)\left(\mathbf{C}_{p}\right)$, let $(h(P), A(P))=(h(E),|\operatorname{Aut}(\bar{E})| / 2)$ if $P$ corresponds to an elliptic curve $E$ with good reduction, $\bar{E}$, and $(h(P), A(P))=$ $(0,1)$ otherwise.

If $r \in \mathbf{R}_{>0}$, let $\mathcal{B}_{r}$ be the annulus $\left\{\kappa \in \mathcal{B}: v\left(w_{p}(\kappa)\right)<r\right\}$.

Conjecture 1.1. (a) The restriction of $\mathcal{E}_{p}$ to $\mathcal{Z}_{p}:=Z \times \mathcal{B}_{c_{p}}$, where $c_{p}=3$ if $p=2$ and 1 otherwise, analytically continues to a function whose absolute value is bounded by 1 on the rigid connected component $\mathcal{V}_{p}$ of

$$
\left\{(P, \kappa) \in X_{1}(\mathbf{q}) \times \mathcal{B}: h(P)<\frac{p}{p+1}, A(P) \cdot h(P)<v\left(w_{p}(\kappa)\right)<c_{p}\right\}
$$

containing $\mathcal{Z}_{p}$ 卢

(b) Moreover, if $\kappa \in \mathcal{B}_{c_{p}}$, the restriction of this function to $\left.\mathcal{V}_{p}\right|_{\kappa} \subset X_{1}(\mathbf{q})$ does not analytically continue to any larger connected region in $X_{1}(\mathbf{q})$.

Theorem 1.2. Conjecture 1.1 is true if $p$ equals 2 or 3.

Proof. We will use the notation $E_{(t, i)}$ for the Eisenstein series of $\S \mathrm{B} 1$ of [Co97i].

We first prove (a). Suppose $p=2$. If $P \in X_{1}(4)=X_{0}(4), A(P)=12$ if $P$ corresponds to an elliptic curve with good supersingular reduction and 1 otherwise.

${ }^{3}$ This, in fact, can be deduced more directly using the arguments which established Corollary 4.1.2 of [Co97i]. The point is, the function labeled there, $e^{s}$, naturally extends to

$$
\mathcal{B} \times \bigcup_{\substack{v \in I_{2}^{*} \\|e-1|_{X(v)}<1}} X(v) .
$$

${ }^{4}$ We use the definition on page 97 of Ka73.

${ }^{5} \mathcal{B}_{c_{p}}$ (for all $p$ ) is the largest annulus of the form $\mathcal{B}_{r}$ containing no points corresponding to classical level 1 forms. 
Let $y_{2}$ be the function on $X_{1}(4)$,

$$
y_{2}=\frac{E_{(2,0)} / V\left(E_{(2,0)}\right)-1}{24} .
$$

As explained in BK05, $y_{2}$ yields an isomorphism $X_{1}(4) \rightarrow \mathbf{P}^{1}$. By Theorem 7 and a remark on page 613 (the end of the proof) of [BK05,

$$
\mathcal{E}_{2} \in \mathbf{Z}_{2}\left[\left[w_{2} / 8,8 y_{2}\right]\right] \cap \mathbf{Z}_{2}\left[\left[w_{2}, y_{2}\right]\right] .
$$

In other words,

$$
\mathcal{E}_{2}=\sum_{j \geq 0}\left(\sum_{i<j} b_{i j}\left(\frac{8}{w_{2}}\right)^{j-i}+\sum_{i \geq j} b_{i j} w_{2}^{i-j}\right)\left(w_{2} y_{2}\right)^{j},
$$

where $b_{i j} \in \mathbf{Z}_{2}$. So $\mathcal{E}_{2}$ continues to and is bounded by one on the connected component $\mathcal{D}_{2}$ of

$$
\left\{(P, \kappa) \in X_{1}(4) \times \mathcal{B}:-v\left(y_{2}(P)\right)<v\left(w_{2}(\kappa)\right)<3\right\}
$$

containing $\mathcal{Z}_{2}$.

By Lemma 2 (iii) of BK05, if $F_{2}=V(\Delta) / \Delta$, which is a modular function of level 2 that yields an isomorphism $X_{1}(2)=X_{0}(2) \rightarrow \mathbf{P}^{1}$, then

$$
F_{2}=\frac{y_{2}\left(1+8 y_{2}\right)}{\left(1-8 y_{2}\right)^{2}} \text {. }
$$

Thus if $v\left(y_{2}\right)>-3,\left|F_{2} / y_{2}-1\right|<1$.

One can show that

$$
\frac{\left(2^{8} F_{2}+1\right)^{3}}{F_{2}}=j .
$$

Now,

$$
v(j(E))=12 h(E)
$$

if $12>v(j(E)) \geq 0$, by Theorem 2.2. So if $v\left(y_{2}\right)>-3$, then $v\left(F_{2}\right)>-3$ and

$$
v\left(F_{2}\right)=-12 h .
$$

This implies $\mathcal{D}_{2}$ is contained in $\mathcal{V}_{2}$.

If $m$ and $n$ are positive integers, let $\pi(m n, n): X_{1}(m n) \rightarrow X_{1}(n)$ be the "forgetful map". The image of $\mathcal{V}_{2}$ in $X_{1}(2)$ is contained in the connected component $\mathcal{C}$ of $\pi(2,1)^{-1}\{x \in X(1): v(j(x))<3\}$ containing the cusp $\infty$ by (4). Also, from (3), we see that $v\left(F_{2}\right)>-3$ on $\mathcal{C}$. Using $(2)$, we see that $v\left(y_{2}\right)>-3$ on the connected component of $\pi(2,2)^{-1} \mathcal{C}$ containing the cusp $\infty \in X_{1}(4)$. Thus $\mathcal{V}_{2}=\mathcal{D}_{2}$.

Now, suppose $p=3$. Let

$$
y_{3}=\frac{E_{(1,0)} / V\left(E_{(1,0)}\right)-1}{6} .
$$

Then $y_{3}$ is a level 9 modular function giving an isomorphism $X_{0}(9) \rightarrow \mathbf{P}^{1}$. By Theorem 4.2 and the proof of Corollary 4.3 of [Ro09,

$$
\mathcal{E}_{3} \in \mathbf{Z}_{3}\left[\left[w_{3} / 3,3 y_{3}\right]\right] \cap \mathbf{Z}_{3}\left[\left[w_{3}, y_{3}\right]\right] .
$$


By Lemma 2.4(3) of [Ro09], if $F_{3}=\sqrt{V(\Delta) / \Delta}$ (a level 3 modular function yielding $\left.X_{1}(3)=X_{0}(3) \cong \mathbf{P}^{1}\right)$, then

$$
F_{3}=\frac{y_{3}\left(1+3 y_{3}+9 y_{3}^{2}\right)}{\left(1-3 y_{3}\right)^{2}}
$$

Thus, if $v\left(y_{3}\right)>-1$, then $\left|F_{3} / y_{3}-1\right|<1$.

As McMurdy pointed out,

$$
j=\frac{\left(1+27 F_{3}\right)\left(1+243 F_{3}\right)^{3}}{F_{3}} .
$$

If $v\left(F_{3}\right)>-3$, then $v\left(F_{3}\right)=-v(j)$. Also, using Theorem 2.2, if $v\left(y_{3}\right)>-1$, we see that $v\left(y_{3}\right)=v\left(F_{3}\right)=-v(j)=-6 h$. We can now argue as above to show $\mathcal{E}_{3}$ extends to and is bounded by one on $\mathcal{V}_{3}$. This proves (a).

To establish the rest of the theorem we will use

Lemma 1.3. If $f$ is an analytic function on the open unit disk $B(0,1)$ and $f$ analytically continues to a strictly larger connected rigid subspace of the closed unit disk $\mathbf{B}^{1}$, then there exists an open affine $U$ in $\mathbf{A}_{\mathbf{F}}^{1}$ containing 0 and an analytic function $F$ on the affinoid $Y=\operatorname{red}^{-1} U$ such that $\left.F\right|_{B(0,1)}=f$. Moreover, if $\|f\|_{B(0,1)} \leq 1$, then $\|F\|_{Y} \leq 1$ and $\overline{A^{o}(Y)}=\mathcal{O}_{\mathbf{A}_{\mathbf{F}}^{1}}(U)$.

Proof. This follows from the fact that any connected affinoid in $\mathbf{B}^{1}$ which properly intersects $B(0,1)$ must contain the complement of finitely many residue disks.

Suppose $p=2$ and suppose $\kappa \in \mathcal{B}$ and $v\left(w_{2}(\kappa)\right)<3$. Then the fiber above $\kappa$ of $\mathcal{V}_{2}$ is isomorphic to $B(0,1)$ and we may regard $X=: X_{\kappa}=w_{2}(\kappa) y_{2}$ as a uniformizing parameter on it. Thus, as pointed out on page 614 of [BK05], there is a $g_{\kappa}(z) \in \mathcal{O}_{\kappa}[[z]]$ such that, after pullback, the restriction of $\mathcal{E}_{2}$ to this fiber equals $g_{\kappa}\left(w_{2}(\kappa) y_{2}\right)$.

It follows that if we take $f=X g_{\kappa}(X)$ and $f$ extends to an analytic function on a larger connected region in the fiber above $\kappa$ of $X_{1}(4) \times \mathcal{B}_{3}$ than $\left.\mathcal{V}_{2}\right|_{\kappa}$, then there must be an open affine $U$ in $\mathbf{A}_{\mathbf{F}}^{1}$ containing 0 and an analytic function $F$ on $Y=\operatorname{red}^{-1} U$ such that $\left.F\right|_{B(0,1)}=f$. Then $\bar{F}$ must be a rational function in $X$. It follows from the analysis on page 614 of [BK05] that

$$
\bar{F}(X)^{2}+\bar{F}(X)+X=0 .
$$

But, this means $\bar{F}$ is integral over $\mathbf{F}[X]$, which implies $\bar{F} \in \mathbf{F}[X]$, but there are no solutions of (5) in $\mathbf{F}[X]$. This implies $\mathcal{E}_{2}$ does not continue to a larger connected region in the fiber above $\kappa$.

Now suppose $p=3$ and $\kappa \in \mathcal{B}$. Then if $v\left(w_{3}(\kappa(4))\right)<1$, by $\S 5$ of [Ro09 there is a $g_{\kappa}(z) \in \mathcal{O}_{\kappa}[[z]]$ such that the restriction of $\mathcal{E}_{3}$ to the fiber above $\kappa$ equals $g_{\kappa}\left(w_{3}(\kappa) y_{3}\right)$. By the proof of Lemma 5.1 of [Ro09], if $G(X)=X^{2} \bar{g}_{\kappa}(X)$, then

$$
G(X)^{3}+G(X)^{2}+X G(X)-X^{3}=0 .
$$

This has no solution in $\mathbf{F}[X]$, so as above $\mathcal{E}_{3}$ does not continue to a larger connected region in the fiber above $\kappa$. This completes the proof of (b). 


\section{Valuation of Hasse}

We use the notation and formulas of [DT75]. Suppose $R$ is a ring, $p=0$ in $R$ and $a_{1}, \ldots, a_{6} \in R$. Let

$$
\begin{gathered}
E: y^{2}+a_{1} x y+a_{3} y=x^{3}+a_{2} x^{2}+a_{4} x+a_{6}, \\
\pi=\mathrm{d} x /\left(2 y+a_{1} x+a_{3}\right)=\mathrm{d} y /\left(3 x^{2}+2 a_{2} x+a_{4}-a_{1} y\right),
\end{gathered}
$$

$b_{2}=a_{1}^{2}+4 a_{2},-4 b_{8}=b_{4}^{2}-b_{2} b_{6}, c_{4}=b_{2}^{2}-24 b_{4}$ and $\Delta=b_{4}^{3}-27 b_{6}^{2}+b_{8}\left(36 b_{4}-\right.$ $\left.b_{2}^{2}\right)$. Suppose $\Delta \in R^{*}$. Then $E$ is an elliptic curve over $\left.R\right]^{6} \pi$ is a non-vanishing differential and

$$
j(E)=c_{4}^{3} / \Delta .
$$

The following lemma should be well known (it follows easily from the definition).

Lemma 2.1. We have $H(E, \pi)=a_{1}$ if $p=2$, and $H(E, \pi)=b_{2}$ if $p=3$.

Theorem 2.2. Suppose $P$ is a point on the $j$-line $X(1)$ over $\mathbf{R}_{p}$ corresponding to an elliptic curve $E_{P}$ with good supersingular reduction and $P_{0} \in X(1)(\mathbf{W})$ such that $\bar{P}_{0}=\bar{P}$ and $j\left(P_{0}\right)=0$ if $p \leq 3$ or $E_{p-1}\left(P_{0}\right)=0$ if $p>37$ Then

$$
h\left(E_{P}\right)=\frac{v\left(j(P)-j\left(P_{0}\right)\right)}{\left|\operatorname{Aut}\left(\bar{E}_{P}\right)\right| / 2}
$$

if either expression is strictly less than one.

Proof. When $p$ is 2 or 3 , this follows from (6), Theorem 10.1 of chapter III of Si86] (which implies $\left|\operatorname{Aut}\left(\bar{E}_{P}\right)\right| / 2=12$ if $p=2$ and 6 if $p=3$ ) and Lemma 2.1. When $p>3$,

$$
H=E_{p-1} \bmod p,
$$

by $\S 2.1$ of Ka73]. Suppose $N \geq 3,(N, p)=1$, and let $E(N)$ denote the universal elliptic curve over $Y(N)$ (the open modular curve of level $N$ ) and $G=\Gamma(1) / \Gamma(N)$. Let $f: Y(N) \rightarrow Y(1)$ be the natural map. Then $G$ acts on $(E(N), Y(N))$ and hence on $\omega=f_{*} \Omega_{E(N) / Y(N)}^{1}$. Now $E_{p-1}$ may be considered a $G$-invariant section of $\omega^{p-1}$. Let $U \subset Y(1)$ be the residue disk of $\bar{P}$ and $V$ one of the residue classes (which are disks over $\mathbf{W}$ ) above $\bar{f}^{-1}(\bar{P})$. Then $f: V \rightarrow U$ is finite, surjective and of degree $d:=\left|\operatorname{Aut}\left(\bar{E}_{P}\right)\right| / 2$. Moreover, if $d>1, U$ will contain a point $R$ with $j(R)$ equal to 0 or 1728 and $\left.f\right|_{V}$ is totally ramified at the unique point $Q$ above $R$. As is well-known, $E_{p-1}(R)=0$ (as $\bar{P}$ is supersingular) 8 so $R=P_{0}$. If $\eta$ is a basis for $\omega(V)$ on the formal scheme attached to $V$, then $\left.E_{p-1}\right|_{V}=s \eta^{p-1}$, where $s: V \rightarrow B(0,1)$ is an isomorphism which vanishes at $Q$. Moreover,

$$
f^{*}\left(\left.j\right|_{U}\right)=j\left(P_{0}\right)+s^{d} g
$$

where $g \in A^{0}(V)^{*}$.

Now, suppose $A \in V\left(\mathbf{R}_{p}\right)$ and $f(A)=P$. Then

$$
j(P)-j\left(P_{0}\right)=s(A)^{d} g(A) .
$$

\footnotetext{
${ }^{6}$ If $p>2, E$ also has the equation $y^{2}=4 x^{3}+b_{2} x^{2}+2 b_{4} x+b_{6}$. (See chapter III, §1, of [Si86].)

${ }^{7}$ It is known that if $p>3$, then $E_{p-1}$ has unique zero in each supersingular disk.

${ }^{8}$ If $\tau$ is in the upper half-plane, $E_{k}(\tau)=\frac{1}{2} \sum_{(a, b)=1} \cdot(a \tau+b)^{-k}$, so if $\tau$ is a quadratic root of unity, then $\tau^{-k} E_{k}(\tau)=E_{k}(\tau)$.
} 
Since $v(g(A))=0$,

$$
v\left(j(P)-j\left(P_{0}\right)\right)=d v(s(A))=\left(\left|\operatorname{Aut}\left(\bar{E}_{P}\right)\right| / 2\right) \cdot h\left(E_{P}\right)
$$

if $v\left(j(P)-j\left(P_{0}\right)\right) /\left(\left|\operatorname{Aut}\left(\bar{E}_{P}\right)\right| / 2\right)$ or $h\left(E_{P}\right)$ is strictly less than one.

Remarks 1. (i) One can show that $h(E)=v(E)$, where $v(E)$ is as defined on page 36 of $\mathrm{Bu} 03$ when $v(E)<p /(p+1)$.

(ii) Proposition 1 of $[\mathrm{BC} 06]$ follows from Theorem 2.2.

\section{REFERENCES}

[Bu03] Buzzard, Kevin, Analytic continuation of overconvergent eigenforms. J. Amer. Math. Soc. 16 (2003), no. 1, 29-55. MR.1937198(2004c:11063)

[BK05] Buzzard, Kevin, and Kilford, L. J. P., The 2-adic eigencurve at the boundary of weight space. Compos. Math. 141 (2005), no. 3, 605-619. MR2135280(2005m:11101)

[BC06] Buzzard, Kevin, and Calegari, Frank, The 2-adic eigencurve is proper. Doc. Math. 2006, Extra vol., 211-232. MR2290588 (2007j:11055)

[Bu07] Buzzard, Kevin, Eigenvarieties, in " $L$-functions and Galois representations", 59-120, London Math. Soc. Lecture Note Ser., 320, Cambridge Univ. Press, Cambridge, 2007. MR2392353 (2010g:11076)

[Co97i] Coleman, Robert, $p$-adic Banach spaces and families of modular forms. Invent. Math. 127 (1997), no. 3, 417-479. MR1431135 (98b:11047)

[Co97ii] , On the coefficients of the characteristic series of the $U$-operator. Elliptic curves and modular forms (Washington, DC, 1996). Proc. Nat. Acad. Sci. U.S.A. 94 (1997), no. 21, 11129-11132. MR1491972 (98m:11032)

[CM98] Coleman, Robert, and Mazur, Barry, The eigencurve. Galois representations in arithmetic algebraic geometry (Durham, 1996), 1-113, London Math. Soc. Lecture Note Ser. 254, Cambridge Univ. Press, Cambridge, 1998. MR.1696469 (2000m:11039)

[DT75] Deligne, Pierre, Courbes elliptiques: Formulaire d'aprés J. Tate. Modular functions of one variable, IV (Proc. Internat. Summer School, Univ. Antwerp, Antwerp, 1972), pp. 53-73. Lecture Notes in Math., 476, Springer, Berlin, 1975. MR0387292 (52:8135)

[Ka73] Katz, Nicholas, $p$-adic properties of modular schemes and modular forms. Modular functions of one variable, III (Proc. Internat. Summer School, Univ. Antwerp, Antwerp, 1972), pp. 69-190. Lecture Notes in Mathematics, 350, Springer, Berlin, 1973. MR0447119 $(56: 5434)$

[La78] Lang, Serge, Cyclotomic Fields, Springer-Verlag, 1978. MR0485768 (58:5578)

[Ro09] Roe, David, The 3-adic eigencurve at the boundary of weight space, to appear.

[Si86] Silverman, Joseph, The Arithmetic of Elliptic Curves, Graduate Texts in Mathematics, 106, Springer-Verlag, New York, 1986. MR817210 (87g:11070)

Department of Mathematics, University of California, Berkeley, California 947203840

E-mail address: coleman@math.berkeley.edu 